ARTICLE

https://doi.org/10.1038/s41467-019-11236-3

\title{
Integrated analyses of murine breast cancer models reveal critical parallels with human disease
}

Jonathan P. Rennhack, Briana To, Matthew Swiatnicki², Caleb Dulak, Martin P. Ogrodzinski1,3, Yueqi Zhang ${ }^{1}$, Caralynn Li ${ }^{1}$, Evan Bylett ${ }^{1}$, Christina Ross ${ }^{4}$, Karol Szczepanek ${ }^{4}$, William Hanrahan ${ }^{1}$, Muthu Jayatissa ${ }^{1}$, Sophia Y. Lunt (iD) ${ }^{3,4}$, Kent Hunter (i) ${ }^{5}$ \& Eran R. Andrechek ${ }^{1}$

Mouse models have an essential role in cancer research, yet little is known about how various models resemble human cancer at a genomic level. Here, we complete whole genome sequencing and transcriptome profiling of two widely used mouse models of breast cancer, MMTV-Neu and MMTV-PyMT. Through integrative in vitro and in vivo studies, we identify copy number alterations in key extracellular matrix proteins including collagen 1 type 1 alpha 1 (COL1A1) and chondroadherin (CHAD) that drive metastasis in these mouse models. In addition to copy number alterations, we observe a propensity of the tumors to modulate tyrosine kinase-mediated signaling through mutation of phosphatases such as PTPRH in the MMTV-PyMT mouse model. Mutation in PTPRH leads to increased phospho-EGFR levels and decreased latency. These findings underscore the importance of understanding the complete genomic landscape of a mouse model and illustrate the utility this has in understanding human cancers.

\footnotetext{
${ }^{1}$ Department of Physiology, Michigan State University, East Lansing, MI 48824, USA. ${ }^{2}$ Department of Microbiology and Molecular Genetics, Michigan State University, East Lansing, MI 48824, USA. ${ }^{3}$ Department of Biochemistry and Molecular Biology, Michigan State University, East Lansing, MI 48824, USA. ${ }^{4}$ Department of Chemical Engineering and Materials Science, Michigan State University, East Lansing, MI 48824, USA. ${ }^{5}$ Laboratory of Cancer Biology and Genetics, National Cancer Institute, National Institutes of Health, Bethesda, MD 20892, USA. Correspondence and requests for materials should be addressed to E.R.A. (email: andrech1@msu.edu)
} 
B reast cancer is an extremely prevalent disease, with an estimated one in eight women experiencing the disease in her life. Owing to its prevalence, it represents a huge public health concern. A hallmark of the breast cancer is its heterogeneity. Heterogeneity in human breast cancer is present in genomic events, gene expression, metastatic potential, and treatment response. Clinically, breast cancer has been classified on the status of estrogen receptor (ER), progesterone receptor (PR), human epidermal growth factor receptor 2 (HER2), as well as proliferative markers such as Ki67. However, with the advancement of transcriptomics and the advent of DNA microarray technology breast cancers began to be profiled on their global genomic profile. Based upon hierarchical clustering of breast cancer patients five subtypes were identified Luminal A, Luminal B, Basal, HER2 positive, and normal-like ${ }^{1,2}$. To identify heterogeneity of the disease from a multi-omic perspective, international efforts have been undertaken to profile breast cancer using multiple -omic platforms ${ }^{3,4}$. These efforts have been productive in identifying a number of genomic events present in breast cancer patients.

To assess gene function in tumor biology, studies have used model systems, including genetically engineered mouse models (GEMMs). However, the genetic landscape of breast cancer mouse models is poorly understood. Recent studies in lung and skin cancers have characterized mouse models of those diseases and have drawn important conclusions about each model ${ }^{5,6}$.

The transcriptional landscape of breast cancer GEMMs, with particular attention paid to relationships with human breast cancer has been extensively studied ${ }^{7-9}$. As follow up to these studies the copy number landscape of the models has been predicted $^{10,11}$. Here, we present whole-genome sequencing data of two highly utilized mouse models of breast cancer, MMTV-Neu ${ }^{12}$ and MMTV-PyMT ${ }^{13}$. In PyMT tumors we identify a highly conserved mutation in the protein tyrosine phosphatase receptor (Ptprh) resulting in elevated EGFR activity and erlotinib sensitivity. In Neu tumors, a copy number alteration including collagen type 1 alpha 1 (Colla1) and chondroadherin (Chad) alters metastatic potential, which is validated through genetic ablation. Altogether, this data demonstrates that genomic alterations beyond the initiating oncogene need to be considered when choosing a model system for breast cancer.

\section{Results}

Transcriptional characterization of mouse model. To characterize the genomic landscape of the MMTV-Neu and MMTVPyMT tumors, we created a tumor database with complete phenotypic characterization, including tumor latency, histology, and metastatic burden (Supplementary Table 1). Representative tumors from this database were selected for whole-genome sequencing and whole-transcriptome profiling by microarray. The analysis pipeline then correlated phenotypic changes with molecular profiling, including transcriptomics and sequence alterations. The resulting genes were then filtered through human breast cancer datasets to ensure relevance to human breast cancer and confirmed with in vitro/in vivo experiments (Fig. 1a). To combine two separate batches of MMTV-PyMT and MMTV-Neu we used Bayesian Factor Regression Methods (BFRM) analysis and visualized with principal component analysis (PCA) (Supplementary Fig. 1). A high degree of transcriptomic diversity both between and within each model was observed in hierarchical clustering (Fig. 1b). Consistent with previous studies, there are similarities in the signaling pathways of MMTV-Neu and MMTV-PyMT models ${ }^{14}$. We saw very little difference in the transcriptional landscape of the models. To minimize the bias potentially introduced by clustering approaches we also used PCA to visualize the transcriptome of the models (Supplementary Fig. 2). As expected, these results demonstrate that heterogeneity correlated with tumor histological subtype rather than tumor model, consistent with recent studies ${ }^{7,15}$.

To identify on a transcriptional level if the mouse models represented human breast cancer tumors, we co-clustered the mouse tumors with TCGA tumors from each molecular subtype (Supplementary Fig. 3). Consistent with previously published data, we observed that the considerable diversity within the mouse model led to a cohort of tumors, which co-clustered with the human disease and a cohort that has a unique transcriptional profile. We specifically identified a cohort of MMTV-PyMT tumors that co-cluster with basal tumors as well as a significant portion of MMTV-Neu and MMTV-PyMT tumors, which have transcriptional profiles that are similar to HER2 + breast cancer. It was hypothesized that these transcriptional differences were driven by genomic changes.

Genomic sequencing of mouse models. Following standard informatic pipelines, the whole-genome sequence was analyzed. In mapping, the location of Neu and PyMT insertion was uncovered. MMTV-Neu is inserted into an intron of FARS2 (Phenylalanyl-tRNA synthetase, mitochondrial) on chromosome 13 and PyMT is in an intron of a RIKEN protein on chromosome 8. To validate bioinformatic calls of single-nucleotide variants (SNVs) and copy number variants (CNVs) we used PCR and quantitative PCR (qPCR), observing a validation rate of $85 \%$ (Supplementary Table 2). Whole-genome sequencing revealed large differences in the genomic landscape of the MMTV-Neu (Fig. 1c) and MMTV-PyMT (Fig. 1d) tumors. The two tumor models had similar numbers of SNVs (Fig. 1e and Supplementary Table 3). However, both models were $\sim 20 \mathrm{x}$ more stable than human breast tumors with 0.049 mutations/megabase in the mouse models in comparison to an average of $\sim 1$ mutation/ megabase in breast cancer ${ }^{16}$. Copy number alterations (Fig. If and Supplementary Table 4) and translocations (Fig. $1 \mathrm{~g}$ and Supplementary Table 5) were more frequent in the MMTV-Neu model relative to MMTV-PyMT.

To identify consistent alterations that were unique to each model or shared across both, we first identified those genes effected by SNVs, CNVs, and translocations that were present in each model (Fig. 2a). Interestingly, this resulted in identification of a number of key signaling proteins, including those within the PI3K, KRAS, and MAPK signaling cascade. In addition to the initiating oncogene, both mouse models were largely seen to be associated with a number of copy number alterations. To test whether these copy number alterations were consistently targeting similar pathways and processes we utilized an overrepresentation analysis (Fig. 2b). This resulted in a number of potentially interesting gene signaling pathways, including consistent alterations to PI3K/AKT/MTOR signaling in the MMTVNeu model and E2F1 in the MMTV-PyMT tumors. Each of these models have been shown in the literature to have high signaling in those respective pathways ${ }^{14}$ and the CNV data may show a potential mechanism for upregulation of these signaling pathway. Interestingly, we noted that both models showed consistent copy number alterations in the MAPK signaling cascade with each individual tumor containing multiple nodes amplified (Fig. 2c).

Copy number alterations in mouse models. Owing to the fact genomic events in each mouse model were dominated by copy number alterations as compared to single-nucleotide changes or translocations, we wanted to compare the mouse models to human HER2 + breast cancer, which is also dominated by copy 
a

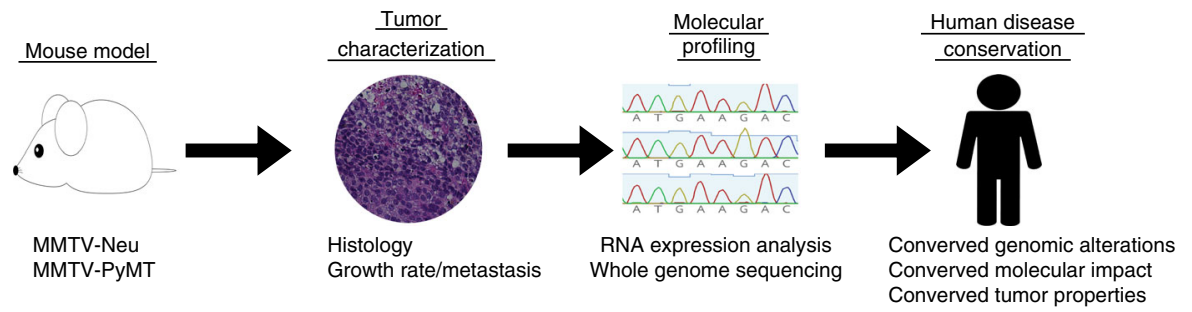

b

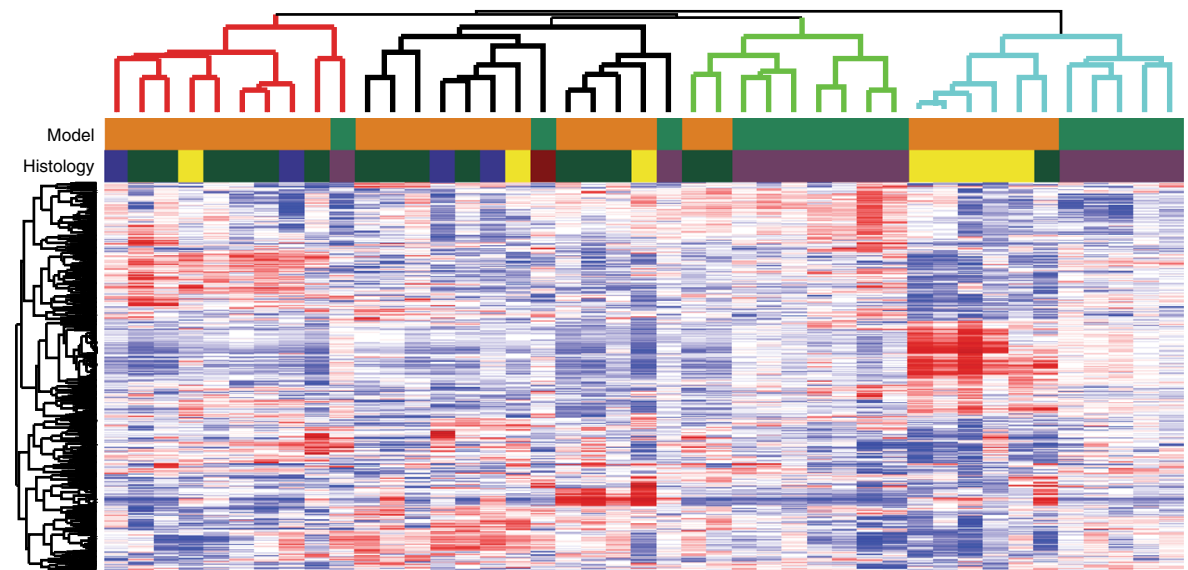

Model MMTV-Neu MMTV-PyMT

Histology $\quad$ EMT MAC $\quad$ PAP $\quad$ SQA $\quad$ CAC

C

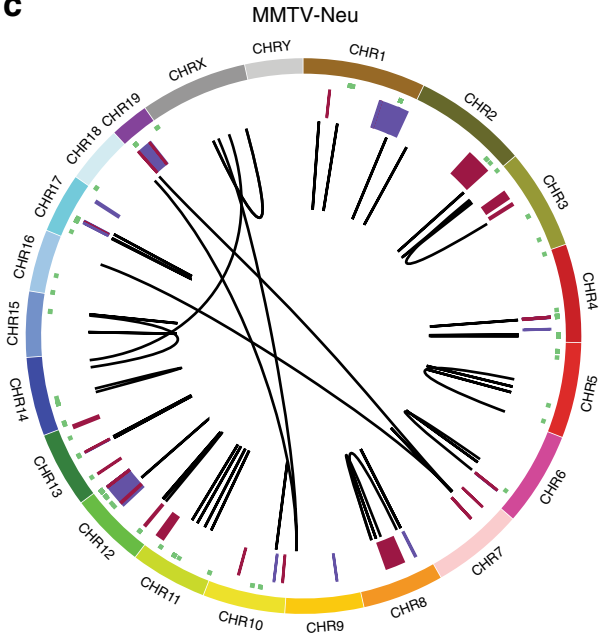

d

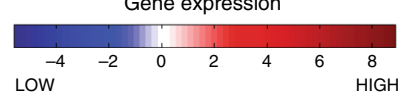

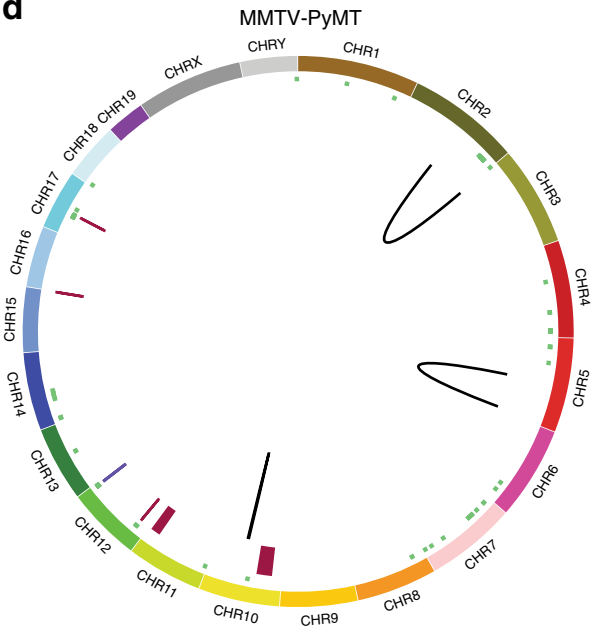

e

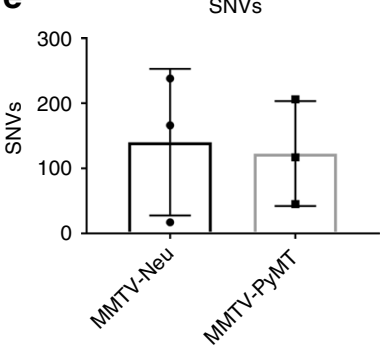

f

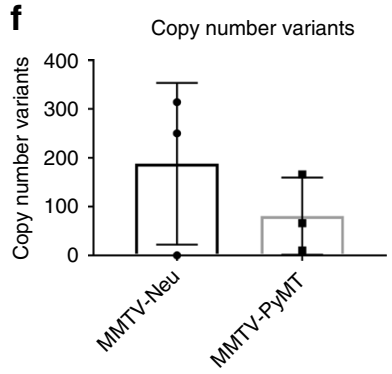



number alterations. Interestingly we saw overlap of partial genomic events but not necessarily the entire event when comparing mouse models to the human tumors. An example of this is the chromosome $12 \mathrm{p}$ amplification in humans, which has been shown to contain the KDM5A amplification as a driver gene. This event was shown to be partially conserved in the mouse model, which shows a large amplification event on chromosome 6 also containing the KDM5A locus (Fig. 3a).

To identify events conserved between mouse models and human breast cancer, we compared amplified and/or deleted 
Fig. 1 Genomic landscape of MMTV-Neu and MMTV-PyMT tumors. The schematic representation of the project workflow is depicted (a), where mammary tumors from two major mouse models are completely characterized through histological, molecular, genomic, and transcriptomic methods. After data integration and analysis, the tumors were compared to human cancers at both genomic and phenotypic levels. Gene expression patterns from MMTV-Neu and MMTV-PyMT tumors were compared by unsupervised clustering, revealing substantial heterogeneity both between and within models. Tumors clustered largely based on histological subtype and not simply genotype. SQU-squamous, MAC-microacinar, PAP-papillary, and CAC-comedoadenocarcinoma ( $n=15$ for MMTV-Neu, $n=25$ for MMTV-PyMT) (b). Circos plots from whole-genome sequencing results for MMTV-Neu (c) and MMTV-PyMT (d) tumors revealed differences between the strains for genomic alterations. Plots display from outside in; Chromosomal location (Each chromosome is unique color), SNVs (green), copy number alterations (Amplification-Red and Deletions-Blue), and translocations (black lines). Variation from multiple tumors is shown for single-nucleotide variants (e), copy number variants (f), and translocations $(\mathbf{g})(n=3$ for each). All error bars present are standard deviation

a



b



C

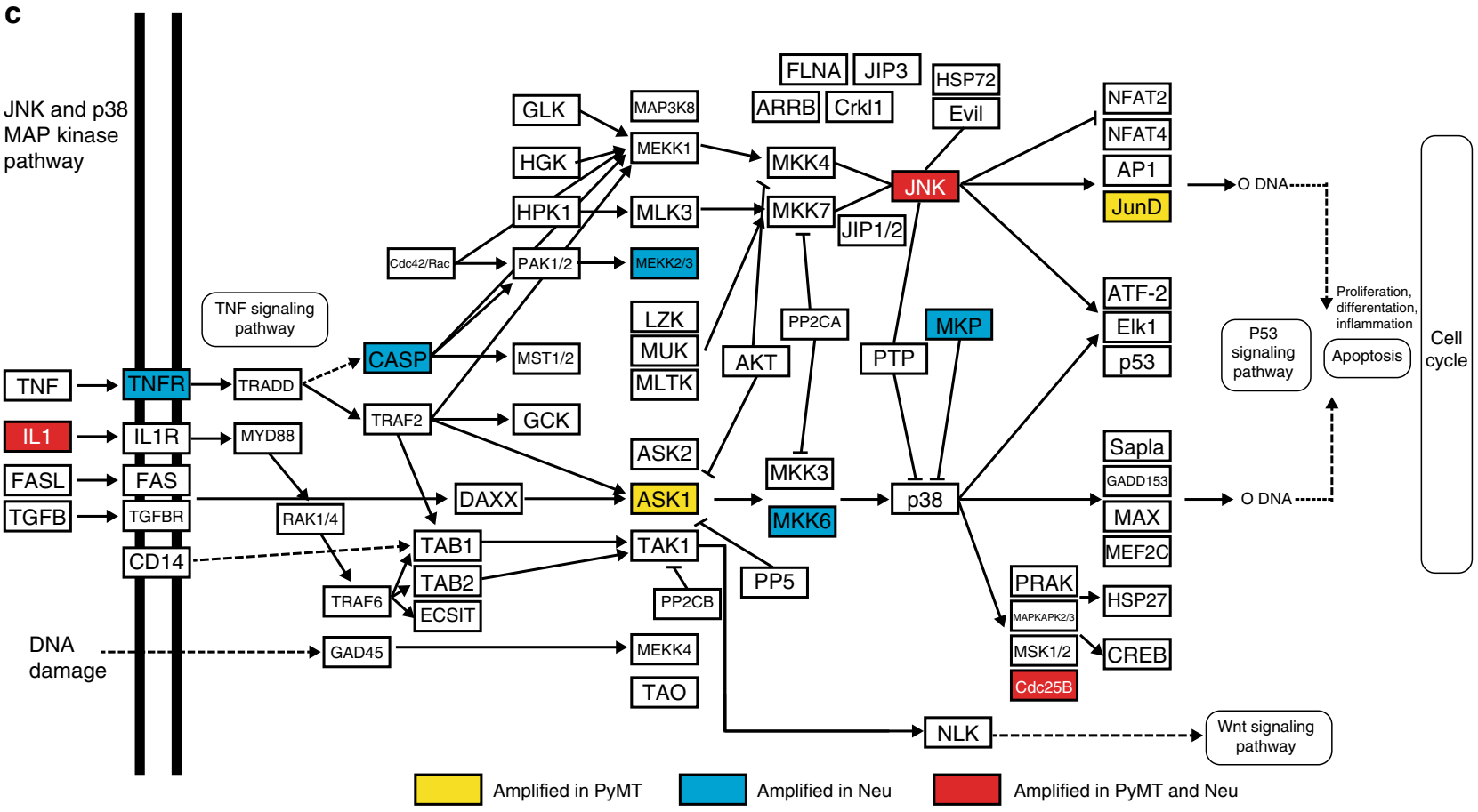

Fig. 2 Signaling pathways are differentially altered in MMTV-Neu and MMTV-PyMT. Key differences in the alterations found in the MMTV-Neu and MMTV-PyMT (a) are apparent in the presence of SNVs (top), CNVs (middle), and translocations (bottom). The presence of copy number variants were apparent in both models. We used over-representation analysis through the assistance of metascape to identify consistently altered genesets (b) and there were important genesets implicated in tumor progression in the copy number profiles of the MMTV-Neu (top), MMTV-PyMT (middle), and both models (bottom). One key gene set over-represented is the MAPK signaling cascade (c) where key signaling nodes can be shown to be altered in each model

genes in each mouse model to those that were amplified or deleted in HER 2 + cancer as identified by the TCGA group (Fig. 3b). While there was significant overlap in both models and the human disease it was surprising that the MMTV-PyMT had a much higher number of amplification and deletion events consistent with the HER2 + samples than the MMTV-Neu samples. To continue to explore the copy number landscape of the MMTV-Neu tumors we filtered to those overlapping with human disease. Two out of the three mouse tumors sequenced did show amplification of driver genes (Fig. 3c). However, these were not consistent across multiple tumors and may not be functionally relevant. 


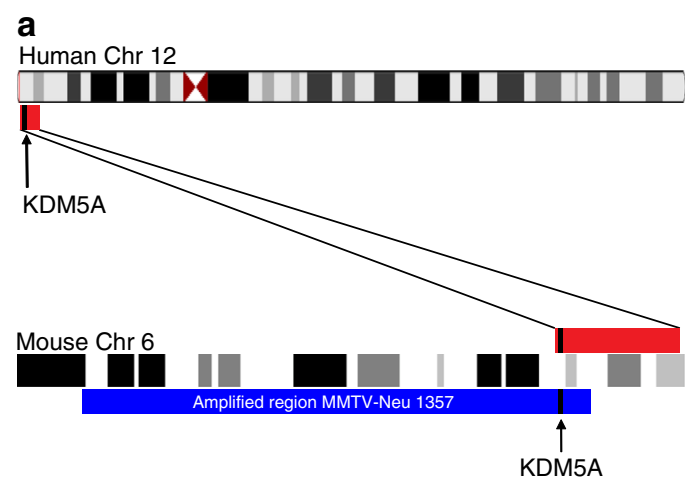

b

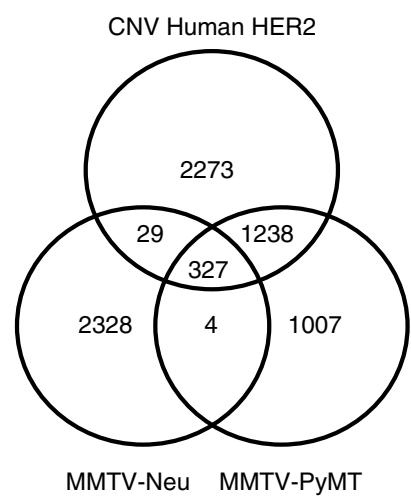

c

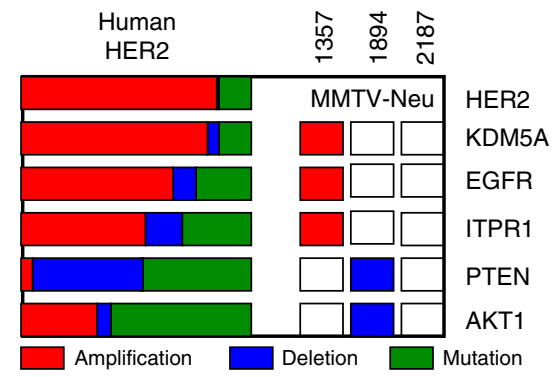

Fig. 3 Copy number profiles of mouse models show similarities with human HER + tumors. Portions of many amplicons are shared between the mouse models and human breast cancer, including the KDM5A amplicon (a). The human amplicon (red, including KDM5A in black) on chromosome 12 consists of numerous genes that are found in the mouse on chromosome 6 (red). The large amplification event in Neu tumors is shown in blue and both amplicons contain KDM5A (a). Consistent overlap between the mouse models and HER2 + human breast cancers was noted (b) with there being more overlap between the MMTV-PyMT and HER2 + model. To determine if these overlapping genes were driving genes, we identified those genes, which were amplified or deleted in the MMTV-Neu tumors and called as driving genes in the TCGA HER2 + cohort (c). CNV (red, amplified and blue, deleted) and mutation status (green) is noted for both human and mouse tumors

Identification of $11 \mathrm{D}$ amplification in MMTV-Neu. To find copy number alteration that are functionally relevant we identified genes, which were consistently amplified or deleted in the mouse models, as well as in human disease. This analysis identified 11 candidate genes, which were highly altered in breast cancer (Supplementary Fig. 4) and predicted to impact tumor biology based upon a literature screen. qPCR gene copy number analysis across an extended tumor panel (15 MMTV-PyMT, 10 MMTV-Neu) identified the rate at which each copy number variant occurred throughout the model (Fig. 4a). This analysis showed that while each of the copy number variants predicted through bioinformatic approaches were valid (Table 2S), the depth of the amplification was largely around 1.5-fold indicating shallow amplification events (Fig. 4b). Interestingly, we identified the largest diversity of copy number profiles in the 11D locus. This locus includes a total of 40 genes, 19 with transcriptomic differences. Depending on the presence or absence of the locus, the tumors exhibited striking differences in structure and behavior. We identified dramatic differences in the tumors with the presence of an 11D amplification with regards to collagen content through a Masson's trichrome (Fig. 4c) stain and the presence of metastatic lesions in the lungs (Fig. 4d).

To identify the driving genes of the metastatic phenotype associated with 11D amplification, we examined human breast cancer for distant metastasis free survival outcomes. We then created CRISPR-Cas9 generated knockouts of two potential metastasis related proteins within the region, Collagen type 1 alpha 1 (Colla1) and Chondroadherin (Chad). Knockouts were generated in two mouse driven tumor cell lines NDL2-5 $5^{17}$ and PyMT $419^{18}$ (Supplementary Fig. 5) and compared to multiple cas9-transfected clones with no sgRNA. NDL2-5 is an 11D amplified Neu driven line, while the 419 line is diploid for the $11 \mathrm{D}$ locus and is driven by PyMT expression. Importantly, we saw no difference in growth rate with the loss of Collal or Chad in our systems (Supplementary Fig. 6). Knockouts of each gene in both cell lines revealed defects in the ability to migrate in a wound-healing assay (Fig. 4e, f) when compared to the nonedited 419 and NDL wild-type clones. Migration was partially rescued with addback of wild-type Collal or Chad, demonstrating that migration defects were not due to off-target effects (Supplementary Fig. 7). Defects in lung colonization in a tail vein injection were also observed with the Collal and Chad knockout cell lines (Fig. 4g, h).

Conservation of Col1a1/CHAD amplification in breast cancer. Mouse chromosome $11 \mathrm{D}$ is conserved in humans and is analogous to chromosomal region $17 \mathrm{q} 21.33$. There is similar amplification event at $17 \mathrm{q} 21.33$, including COL1A1 and $C H A D$ that occurs in $8 \%$ of breast cancer patients. Array CGH from the TCGA data $^{3}$ demonstrates that COL1A1/CHAD amplification was distinct from HER2 amplification (Fig. 5a). Importantly, this amplification is subtype specific; $25 \%$ of Her $2+$ breast cancers have a co-amplification of the $17 \mathrm{q} 21.33$ region along with the HER 2 amplicon while only $6 \%$ of Luminal A, $7 \%$ of Luminal B, and $1.2 \%$ of Basal breast cancers have amplification (Fig. 5b). To investigate the transcriptional impact of the amplification event, we used weighted gene correlation network analysis ${ }^{19}$. This identified a robust transcriptional signature that differentiated COL1A1/CHAD-amplified, Her2-positive tumors from Her2positive tumors without the amplification event (Supplementary Table 6). Unsupervised hierarchical clustering readily identified separation of the two HER2-positive subtypes based on this signature (Fig. $5 \mathrm{c}$ ). These correlated genes were used in a predictive signature to correlate patient outcome with predictive amplification status (Supplementary Fig. 8), revealing that metastasis was associated with the amplification event (Fig. 5d).

To test whether COL1A1 and CHAD were driving the metastasis phenotype in human breast cancer, we used CRISPRi ${ }^{20}$ to create a pooled population of COL1A1 and CHAD knockdown in the Her2 amplified, COL1A1/CHAD amplified breast cancer line BT-474. These knockdowns were shown to have a reduction in CHAD (Supplementary Fig. 5G) and Colla1 (Supplementary Fig. 5F), respectively, as well as the inability to create collagen fibers as identified through a Masson's trichrome (Supplementary Fig. 5G). While we saw no differences in the proliferation rate of these lines in vitro, these knockdowns 
a
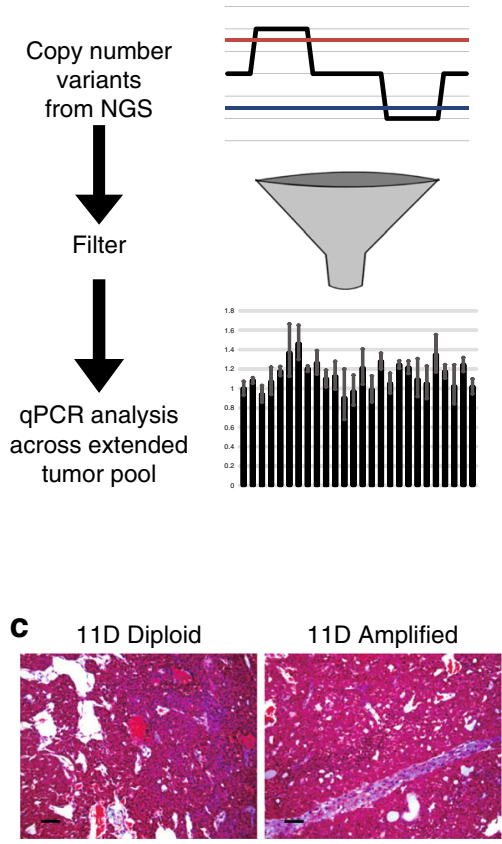

e

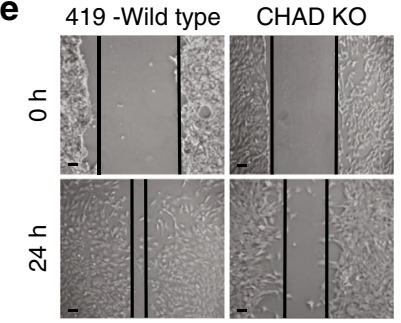

g

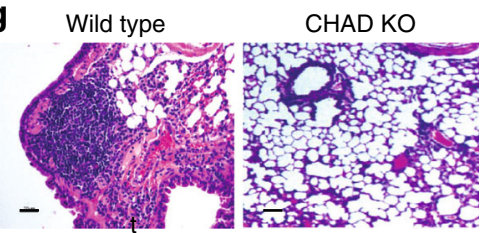

b Fold change


f

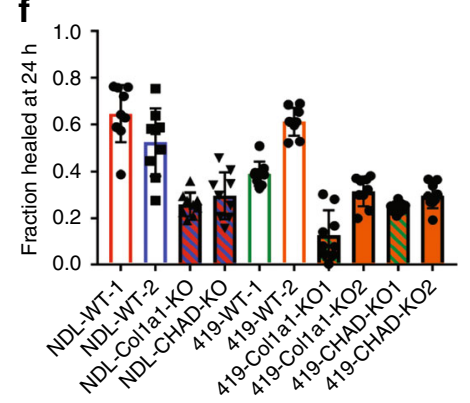

$\mathbf{h}$

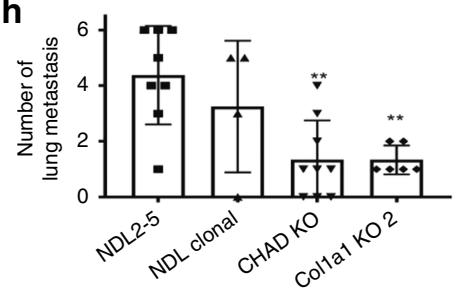

Fig. 4 Copy number alterations alter metastatic potential. Schematic representation of filtering of copy number variants (a) where copy number variants were detected from NGS and filtered to the top 11 variants by selecting those genes that encompassed both mouse models and were conserved in human breast cancer. These genes were then assayed using qPCR analysis across a larger panel of MMTV-Neu $(n=10$, green triangles) and MMTV-PyMT ( $n=$ 15 , red circles) tumors and depicted using a circos plot for genes in chromosomes $2,6,11$, and 17 (b). Heterozygous deletion on the interior of the plot to a threefold amplification on the exterior of the plot is shown. A key copy number alteration in the 11D region encompassing the Colla1 gene (boxed) was observed to correlate with reduction and lack of collagen alignment in Masson's trichrome staining (c) and an increase in metastases in the lungs of mice with Colla1 amplification in the primary tumors (d). CRISPR-Cas9-mediated knockout of two key genes within this region, Colla1 and Chad, show defects in wound healing $(\mathbf{e}, \mathbf{f})($ scale bar $=100 \mu \mathrm{m})(n=9)$ (Colors denote significance by students two-tailed, unpaired $t$-test, $P<0.01$, vs. the same colored control). Knockout also impaired the ability to colonize the lung through a tail vein injection ( $\mathbf{g}, \mathbf{h})(\mathrm{NDL} 2-5 n=12, \mathrm{NDL} 2-5 \mathrm{Clonal} n=4, \mathrm{Chad} \mathrm{KO} n=9$, Col1a1 $\mathrm{KO} n=6$ ). ( ${ }^{\star \star}=P<0.01$, students two-tailed, unpaired $t$-test, for $\mathbf{h}$ ). Scale bar of $20 \mu \mathrm{m}$ displayed on histological image for reference. All error bars denote standard deviation

showed a decreased ability to migrate in a wound-healing assay (Fig. 5e, f). When injected into the mammary fat pad we saw no difference between the WT BT-474 ddcas9 population and the COL1A1 or CHAD knockdown populations in their ability to form tumors. This take rate was consistent with previously published rates ${ }^{21}$. We also saw no difference in growth rates with wild-type and knockdown population (Supplementary Fig. 6B) consistent with the in vitro growth rate data. Importantly, the knockdown lines were unable to metastasize to the lung after being injected into the mammary fat pad (Fig. 5g, h). Altogether these data underscore the importance of identifying copy number variation in mouse models of cancer. 
a

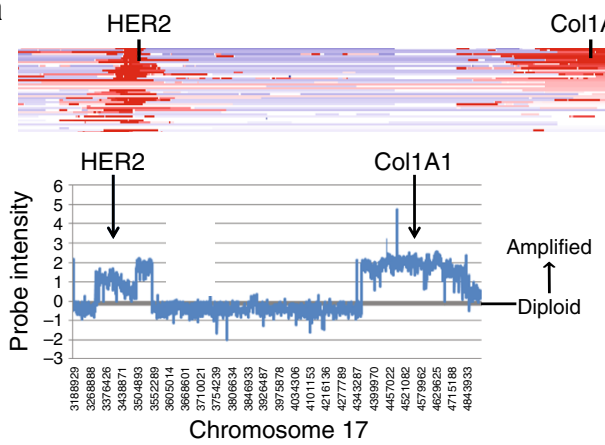

C

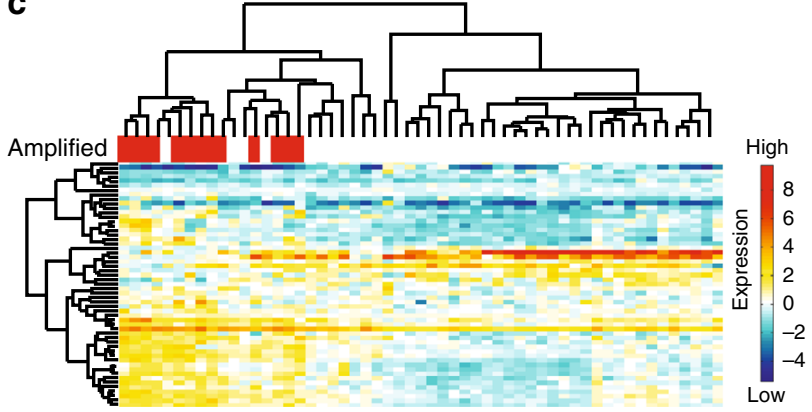

17q21.33 Amplified

e

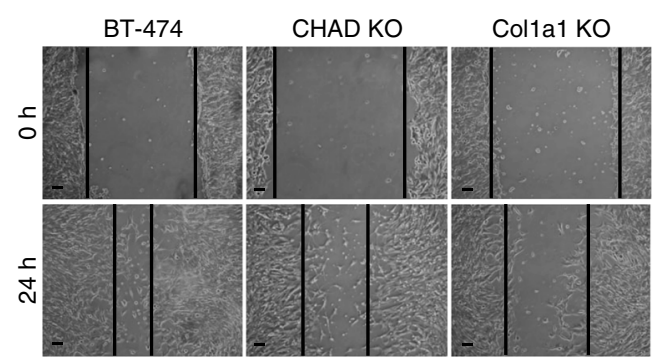

g



b

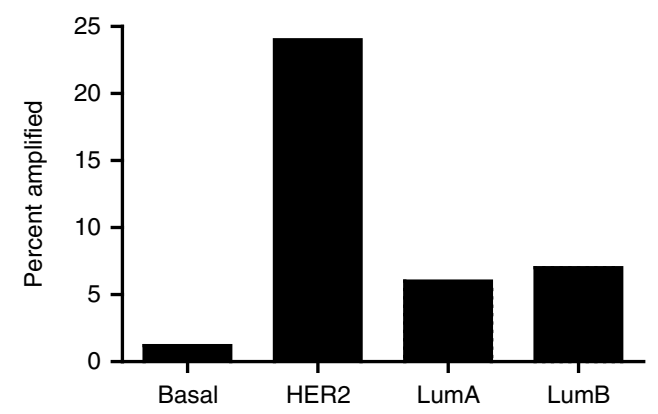

d
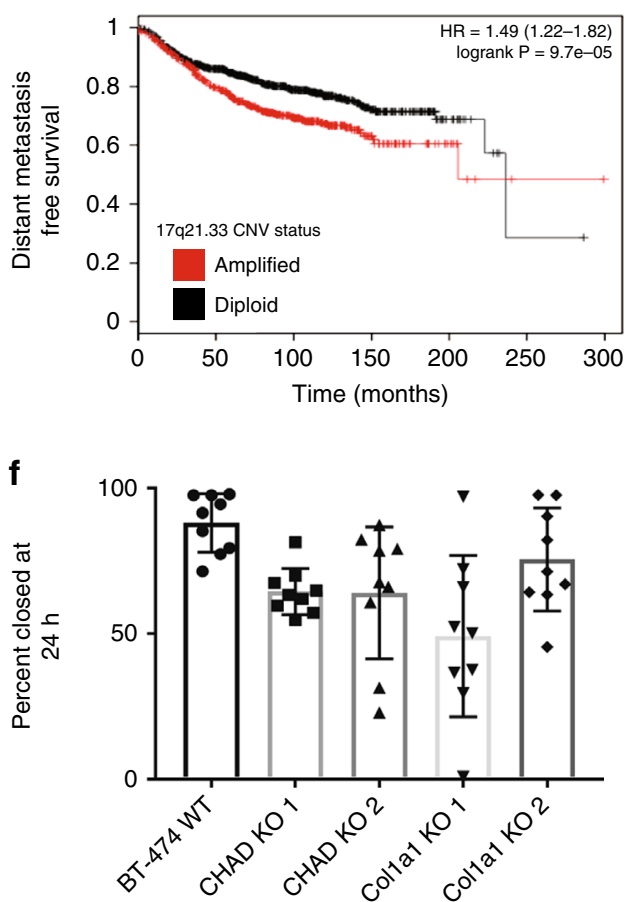

h

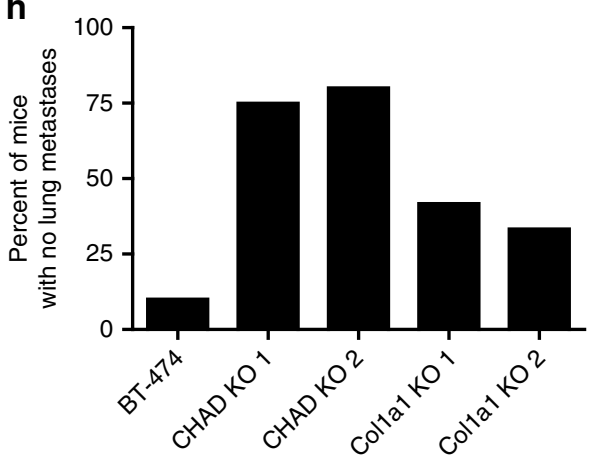

Fig. $511 \mathrm{D}$ amplicon presence and function is conserved in human breast cancer. TCGA breast cancer copy number dataset analysis revealed coamplification of HER2 and the COL1A1 locus through a heatmap across chromosome 17 of multiple samples with each row representing an independent patient sample (a-top) with red representing amplification and blue representing deletion. The COL1A1 amplification event occurred independently of HER2 (A-bottom) as identified by probe intensity of aCGH data of a single TCGA breast cancer patient. The COL1A1/CHAD amplification event was disproportionately found in HER2-positive tumors and is present in $~ 25 \%$ of Her2-positive tumors (b). Gene expression of HER2 + samples with and without the 17q21.33 amplification demonstrated a unique gene expression profile as identified by unsupervised hierarchical clustering (c) and significantly worse overall survival within the KMplotter dataset $(P<0.001$, log rank test) (d). CRISPRi-mediated knockdown of CHAD and COL1A1 in human cell line BT-474 resulted in defects in wound healing $(\mathbf{e}, \mathbf{f})\left({ }^{*}=P<0.05\right.$, students two-tailed, unpaired $t$-test, $\left.n=9\right)$ and distant metastasis to the lung after orthotopic injections ( $\mathbf{g}, \mathbf{h} n=10$, for WT, $n=4$ for CHAD KO1, $n=5$ for CHAD KO2 $n=12$ for Col1a1 KO1, $n=6$ for Col1a1 KO2). Scale bars of 10 $\mu$ m (e), $5 \mathrm{~mm}$ ( $\mathbf{g}$ top), and $20 \mu \mathrm{m}$ ( $\mathbf{g}$ bottom) displayed on image for reference. All error bars presented are standard deviation 
Mutational landscape of mouse models. In addition to copy number alterations, the whole-genome sequence data resulted in the identification of numerous mutations (Fig. 1c-e). When Catalog Of Somatic Mutations In Cancer (COSMIC) mutational signatures ${ }^{22,23}$ were applied to the models, it was observed that the tumor models had similar mutational processes (Supplementary Fig. 9). The MMTV-Neu and MMTV-PyMT tumors both contain the same trinucleotide context of their mutation spectrum. The mutation spectrum shows all nucleotide substitutions present with a slight bias towards $\mathrm{C} / \mathrm{T}$ and $\mathrm{T} / \mathrm{C}$ transitions. When compared to the human mutational signatures, the mutational processes present in both mouse models closely resembles COSMIC signature 5 (Fig S9C). This signature has been shown to be present in breast cancer patients with disease associated with late onset ${ }^{24}$, indicating a similar mutational process in both the human disease and mouse models

Distribution of SNVs reflected patterns seen in the transcriptional data (Fig. 1b) with some events shared between Neu and PyMT tumors while others were unique to the models. Considerable SNV diversity within a model was also prevalent. For instance, the MMTV-Neu model had no genes with shared mutations in all samples and only five genes containing a coding, non-synonymous mutation in more than one sample (Supplementary Fig. 10). Notably we identified mutations within Mucin 4 (Muc4), which are potentially impactful due to Muc4's emerging roles in Her2-positive cancer and metastasis ${ }^{25}$. Interestingly, we observed that PyMT-induced tumors had more SNVs in the coding regions of the genome. Specifically, these mapped to 34 genes, 9 of which overlapped with Neu tumors. A number of genes with coding mutations specifically in PyMT tumors, including Matn2, Plekhm1, Muc6, and Ptprh were observed. Matn226, Plekhm1 ${ }^{27}$, and Muc6 ${ }^{28}$ have all been demonstrated to have roles in tumor progression and metastasis and may contribute to the high metastatic capacity of the MMTV-PyMT model.

To test the frequency of these coding mutations in the models as a whole, we selected a population of $10 \mathrm{MMTV}$-Neu tumors and 15 MMTV-PyMT tumors for targeted resequencing. From these tumors we extracted genomic DNA and performed PCRbased amplification followed by Sanger sequencing of Matn2, Plekhm1, and Ptprh. While Matn2 and Plekhm1 confirmed the whole-genome sequencing variant calls in the sequenced tumors, additional mutations were not found.

Identification and characterization of Ptprh mutation. Strikingly, Ptprh was found to be mutated in $81 \%$ of MMTV-PyMT tumors. Furthermore, the Ptprh mutation was shown to be homozygously mutated in $21 \%$ of PyMT tumors and heterozygously mutated in $60 \%$ of PyMT tumors (Fig. 6a, b). Surprisingly, an identical $\mathrm{C}$ to $\mathrm{T}$ mutation was observed in each tumor resulting in a valine residue being converted to a methionine at amino acid 483 (V483M). To test for the conservation of mutations of Ptprh in mouse strains beyond FVB/NJ, we sequenced Ptprh of MMTV-PyMT models in a C57/Bl6, C57/Bl10, CAST, and MOLF backgrounds, as well as a different inbred MMTVPyMT FVB/NJ line. This analysis showed consistent mutation in the structural fibronectin domains (FN3) and the phosphatase domain of Ptprh (Fig. 6c). Interestingly, we found that the two FVB models contained different mutational patterns, indicating an impact of environmental and potential epigenetic causes of mutational hotspots.

Given that recent work identified the target of PTPRH as EGFR $^{29}$, we hypothesized that EGFR was not dephosphorylated with Ptprh mutation. Testing this, we observed that the V483M mutation correlated with pEGFR levels (Fig. 6d, e). With the resulting increase in EGFR activity, we also observed a significant decrease in tumor latency (Fig. 6f). With an increase in EGFR activity, it was possible that tumors with mutant Ptprh would be dependent upon EGFR signaling. To test this prediction, cell lines derived from Ptprh wild-type and mutant tumors were treated with EGFR targeted therapy. After $48 \mathrm{~h}$, tumors containing Ptprh mutations were shown to be more sensitive to erlotinib treatment (Fig. 6g).

Given the role of EGFR in lung cancer, we next sought to determine if there was a non-EGFR mutant patient population within lung cancer that could benefit from EGFR inhibition. Examination of the pan-lung TCGA data revealed 5\% of patients with a mutation in PTPRH. Importantly, these mutations were shown to be mutually exclusive from EGFR, indicating that patients were likely not treated with EGFR tyrosine kinase inhibitors. To confirm the impact of PTPRH mutations on EGFR activity in human lung tumors we used gene set enrichment analysis to predict EGFR activity of each mutant PTPRH sample. This analysis revealed four key hotspots of mutations driving high EGFR activity, including three in the FN3 domains and one in the phosphatase domain of PTPRH (Fig. 6i)

\section{Discussion}

Altogether, these data emphasize the heterogeneity within tumor models and the importance of understanding the genomic landscape within the tumors. Here, we presented a proof-of-concept study to identify a number of events that have influenced key tumor phenotypes, including metastasis and tumor latency. Through the analysis we have also been able to identify similarities between the mouse models and the human disease from an $\mathrm{SNV}, \mathrm{CNV}$, and translocation standpoint. Despite the limitations with the number of samples, this study offers a unique opportunity to identify genomic alterations which impact tumor behavior and treatment response. These findings have direct therapeutic impact with a potential impact on patient therapeutic intervention and metastatic progression of their disease and underscore the important role genetically engineered mouse models have in understanding tumor biology.

The presence of the Col1A1/CHAD amplification event in the mouse model mirrors the $25 \%$ of human HER 2 + ve breast cancers that also had amplification of a structurally conserved region. Given the potential role for these genes in metastasis, this work clearly indicates that the MMTV-Neu system is an appropriate model for select facets of HER2 tumor biology, including the additional amplification event. However, the lack of amplification of genes surrounding erbB2 in the mouse model indicates that other models with erbB2 amplification ${ }^{30}$ may be more suitable for other studies.

In the PyMT model system, tumor onset is rapid with mice developing tumors 45 days after birth. Despite this rapid onset, the data presented here indicates that over $80 \%$ of PyMT tumors acquire the identical mutation in Ptprh, suggesting that there is a significant evolutionary pressure applied during the initial transformation. Given that the Ptprh mutant does not dephosphorylate EGFR, this results in unchecked activation of a key signaling pathway. In part, this event may impact metastatic progression of this model system. The most likely impact from this discovery will be in the identification of additional tumor patients that may benefit from EGFR TKI therapy.

Taken together, this manuscript provides a resource for investigators to determine how well the subtype of cancer they examine is represented by MMTV-Neu and MMTV-PyMT mouse model systems. While we have explored two genomic events, others of interest are noted and their impact will be elucidated. The data from these two models also underscores the importance of a complete characterization of GEMMs for human cancer. 
a

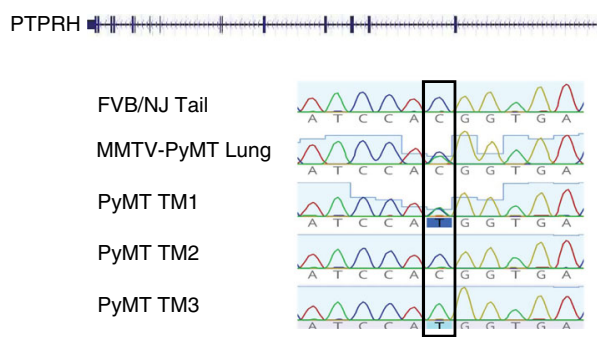

b



Wild type PTPRH

C-T Heterozygous mutation

C-T Homozygous mutation

C

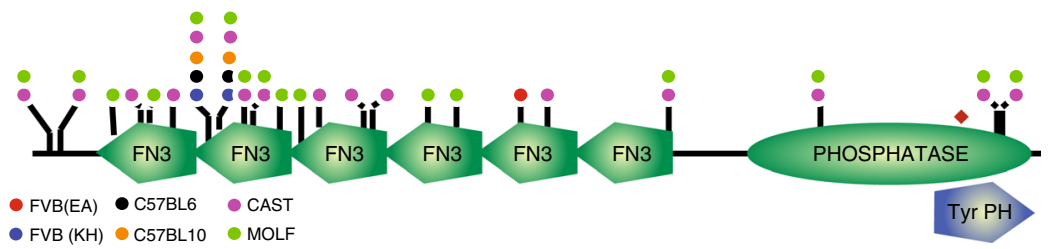

d

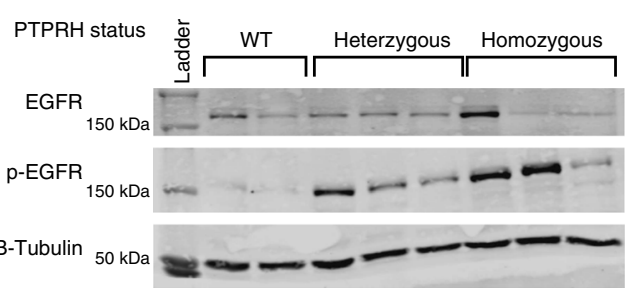

e

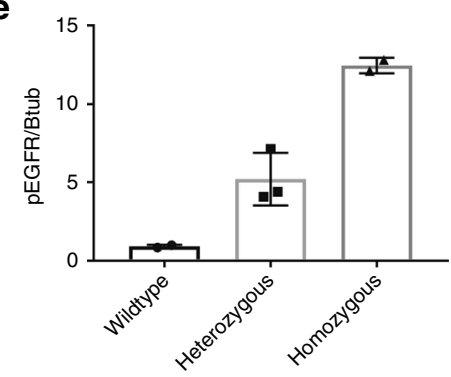

f

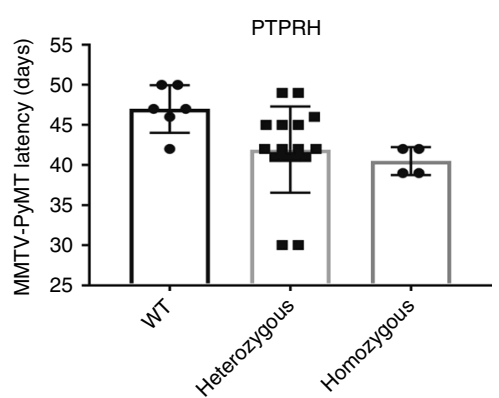

g

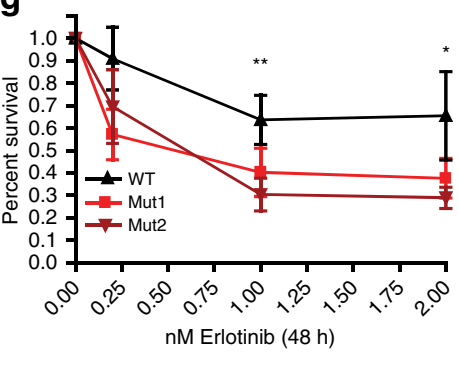

h
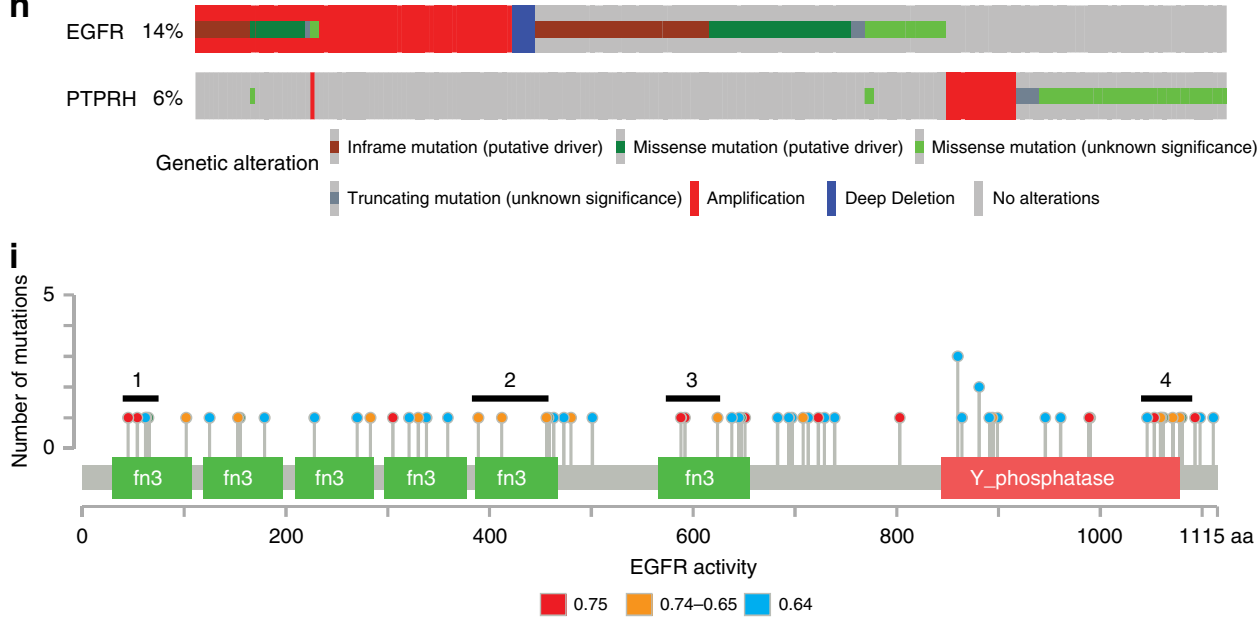

\section{Methods}

Animal studies. All animal husbandry and use was conducted according to local, national, and institutional guidelines. The study received ethical approval from the Michigan State University Institutional Animal Care \& Use Committee (IACUC) under AUF 06/18-084-00. The MMTV-Neu ${ }^{12}$ and MMTV-PyMT ${ }^{13}$ mice were in the FVB background. MMTV-PyMT634 and MMTV-Neu mice were obtained from The Jackson Laboratory. Mice were monitored twice weekly for tumor initiation and growth. At a $2000 \mathrm{~mm}^{3}$ endpoint, mice were necropsied. For mice with multiple tumors the endpoint was established when the primary tumor was at $2000 \mathrm{~mm}^{3}$. Tumors and lungs were collected for genomic analysis, hematoxylin and eosin staining for histological subtyping and presence of pulmonary metastases. The number of metastasis was quantified using a single cut through the lung and count of the number of micro-metastases in that plane. Masson's trichrome staining was used to examine tumors for collagen deposition using standard methods. 
Fig. 6 PTPRH mutations are conserved in MMTV-PyMT and human lung cancer. Phosphotyrosine receptor, Ptprh was shown through Sanger sequencing to have C-T mutations in the MMTV-PyMT tumors while tail and lung samples were normal (a). Expanding targeted sequencing to additional samples revealed a conserved heterozygous mutation in 60\% and homozygous mutation in 21\% (b) of MMTV-PyMT tumors $(n=45)$. Sequencing revealed multiple mouse backgrounds (FVB, C57BL6, C57BL10, CAST, and MOLF) have varied mutations clustered in the functional domains of the Ptprh proteins (c). Increases in pEGFR signaling (d, e) and a decrease in tumor latency $\left(F,{ }^{\star}=P<0.05\right.$, students two-tailed, unpaired $t$-test) was correlated with the mutant Ptprh allele (V483M) within the FVB background. Cell lines derived from Ptprh mutant (V483M) PyMT tumors showed an increased response to EGFR targeted therapy including erlotinib $\left(\mathbf{g}, n=3,{ }^{\star \star}=P<0.01,{ }^{\star}=P<0.05\right.$, students two-tailed, unpaired $t$-test). In human lung cancer (TCGA-pan-lung cancer) $5 \%$ of patients have a mutation in PTPRH, which are mutually exclusive from EGFR (h). High EGFR activity, as determined by gene set enrichment analysis, is associated with mutations clustered within the four indicated structural and functional domains of PTPRH as seen by the colors in the lollipop plot for EGFR activity (i). All error bars presented are standard deviation

Whole-genome sequencing. Flash frozen tumor pieces were ground, and DNA was extracted with the Qiagen Genomic-tip 20/G with the manufacturer's protocol. DNA was sequenced to a depth of 40x with paired end 150 base pair reads on an Illumina HiSeq 2500 using the Illumina TruSeq Nano DNA library preparation.

Transcriptomic profiling. Transcriptome data for this study was previously published $7,31,32$. Affymetrix expression console was used to normalize each individual dataset using RMA normalization. To remove batch effects between datasets BRFM normalization ${ }^{33}$ was performed with standard parameters and visualized with PCA.

Clustering. Unsupervised hierarchical clustering was performed using Cluster 3.0 and the Broad institute's Morpheus interface. Heatmaps were created using the MATLAB imagesc function.

Variant calling. Generated.fastq files were assessed for quality control using FASTQC analysis (http://www.bioinformatics.babraham.ac.uk/projects/fastqc). Reads were trimmed for quality using Trimmomatic ${ }^{34}$. After trimming, data was reassessed for quality using FASTQC. Then reads were aligned to the mm10 mouse reference genome using BWA-mem ${ }^{35}$. After alignment, base recalibration and pcrinduced biases were removed using PICARD tools (http://broadinstitute.github.io/ picard). For variant calling we utilized four software packages, GATK ${ }^{36}$, Mutect $2^{37}$, Strelka $^{38}$, and SomaticSniper ${ }^{39}$. To be a legitimate variant we filtered to only those variants called by 3 of the 4 packages. To control for differences in the FVB strain and the mm10 reference genome we used previously published normal FVB tissue (ERR046395) $^{40}$. To call copy number and structural variants we used Delly ${ }^{41}$. For copy number we used default quality control settings and only analyzed those copy number events, which had precise boundaries and were larger than $100 \mathrm{~KB}$. For translocations we used default quality control setting and precise breakpoints.

Variant verification and extended tumor panel sequencing. For verification of SNVs we used PCR (primers in Supplementary Data)-based amplification followed by Sanger sequencing. For validation of CNVs, we used qPCR (primers in Supplementary Data) on the genomic DNA with the Quantabio PerfeCTa SYBR green kit under the manufacturer's specifications. Primers for PCR and sequencing are listed in Supplementary Data.

Circos visualization. Representative MMTV-Neu and MMTV-PyMT samples were chosen to be displayed as CIRCOS ${ }^{42}$ plots. CIRCOS plots were generated using CIRCOS v 0.69 and SNVs, CNVs, and translocations were mapped according to their location on the mm10 genome.

Mutation signatures. Owing to the low mutational burden of MMTV-Neu and MMTV-PyMT tumors, mutations were combined into a signal analysis for each model. These samples were processed with MutSpec-NMF ${ }^{43}$ for trinucleotide context and comparison to the known human mutation signatures.

Cell lines. The PyMT 419 cell lines were a gracious gift from Dr. Stuart Sell and Dr. Ian Guess ${ }^{18}$. The NDL2-5 cells lines were obtained as a gift from Dr. Peter Siegel ${ }^{17}$. The BT-474 cell line was obtained from Dr. Kathy Gallo and validated using STR fingerprinting analysis performed at Michigan State University. STR fingerprinting was performed with ProMega Cell ID System run on the ABI 3730xl.

CRISPR generated knockouts of PyMT 419 and NDL2-5. CRISPR/Cas9 constructs were created to knockout Collal and Chad in PyMT 419 and NDL2-5. Guides (listed in Supplementary Data) were designed and inserted into Px458, obtained from addgene (Addgene \#48138) as a gift from Feng Zhang, using the BBSI insertion site ${ }^{44}$. Cells were sorted using FACS technology into single cells and grown into clonal population, then screened for the presence of INDELs using Sanger sequencing. Knockouts were further confirmed for the NDL2-5 lines using western blot. Guide Sequences are listed in Supplementary Data. Control lines were transfected with an empty (no sgRNA) and flow cytometry sorted to single-cell clones.

CRISPRi generated knockdowns in BT-474. Knockdowns of Colla1 and CHAD were created in the BT-474 line using CRISPRi technology. Genomic RNA (gRNA) (listed in Supplementary Data) were cloned into a plasmid containing the gRNA under the control of the U6 promoter (Addgene plasmid \#60955) ${ }^{45}$. Lenti virus was created for stable expression of this plasmid and the stable expression of KRABCas9 fusion protein (Addgene plasmid \#60954) ${ }^{45}$. Cells were infected with KRABCas 9 expression virus first and selected for uptake by puromycin treatment. The stable KRAB-Cas9, BT-474 line was then infected with the virus for stable selection of the gRNA for CHAD or COL1A1. These were then sorted using flow cytometery for RFP expression into a pooled population and validated knockdown through western blot. The populations were also assayed for the ability to create functional collagen fibers through a Mason's trichrome. The plasmids used in the part of the project were obtained through Addgene as a gift from Jonathan Weissman.

Wound-healing assay. Wound-healing assays were performed similarly for all cell lines in the manuscript. Cells were grown to $100 \%$ confluence in a six well plate then a wound was created in the middle of the plate. Cells were allowed to close the wound for $24 \mathrm{~h}$ in the presence of Mitomycin $\mathrm{C}$ growth inhibitor then the cells were imaged. Images were quantified for the amount of migration into the wound using ImageJ.

Tail vein injection. NDL2-5 Chad and Colla1 knockout cell lines were injected into the tail vein of syngeneic FVB/NJ mice. Cells were suspended in phosphate buffered saline (PBS) in a single-cell population and injected in a single bolus of $1 \times 10^{6}$ cells in $50 \mu \mathrm{L}$. Mice were monitored for 9 weeks then euthanized. At this point, lungs were collected and stained with Hematoxylin and Eosin to identify the presence of pulmonary metastases.

Mammary fat pad injection. NDL2 $-5 \mathrm{WT}$ and cell lines were suspended in $50 \mu \mathrm{L}$ $\mathrm{PBS}$ and injected into mammary gland number four in syngeneic FVB/NJ mice as a single bolus of $1 \times 10^{6}$ cells. The mice were monitored twice weekly until tumors reached an endpoint of $2000 \mathrm{~mm}^{3}$

BT-474 wild-type and CHAD/COL1A1 knockout lines were suspended in a 1:1 concentration of matrigel:PBS mixture $(50 \mu \mathrm{L})$ and injecting into the mammary gland number four in a single bolus of $1 \times 10^{6}$ cells. Balb/C nude mice were used for these studies. Tumors were monitored until a size of $1000 \mathrm{~mm}^{3}$. Tumors were then resected, and mice were monitored for an additional 4 weeks. At necropsy lungs were imaged for RFP using the IVIS imaging system and then processed for hematoxylin and eosin staining.

Human dataset usage. All human datasets used in this study are publicly available and noted as used in the manuscript. For genomic alteration frequency, the TCGA Breast cancer ${ }^{3}$ and the TCGA-pan-Lung cancer ${ }^{46}$ datasets were used. For the expression-based survival data the KMPlot.com dataset ${ }^{47}$ was used.

Western blotting. Western blots in this manuscript were completed under manufacturer's specifications. Blocking was performed for $1 \mathrm{~h}$ by incubation at room temperature with the LiCor blocking reagents. Western blots were imaged using the LiCor system. The following antibodies were used: COL1A1 [1:1000] (Origene TA309096), CHAD [1:2500] (Abcam ab104757), EGFR [1:1000] (CST D38B1), pEGFR [1:1000] (Invitrogen PA5-37553), HSP90 [1:1000] (CST 4874S), Beta-tubulin [1:1000] (CST 2128S), anti-rabbit secondary [1:10000] (Licor 926-32211), anti-mouse secondary [1:10,000] (Licor 926-68070).

Erlotinib sensitivity assay. Cell lines derived from Ptprh mutant and wild-type tumors were seeded at a concentration of 250 cells $/ \mathrm{mL}$ and subjected to erlotinib treatment for $48 \mathrm{~h}$ with the concentrations stated in the manuscript. Eroltinib was purchased from Cayman Chemical. After treatment with erlotinib or DMSO control, cells were given fresh media to grow for 7 days. Cells were then fixed and stained with crystal violet for counting. 
Statistical considerations. Except where otherwise noted all statistical comparisons are performed with an unpaired students two-tailed, unpaired $t$-test. All error bars presented are standard deviation.

Reporting summary. Further information on research design is available in the Nature Research Reporting Summary linked to this article.

\section{Data availability}

The fastq sequencing datasets for the MMTV-Neu and MMTV-PyMT models has been deposited in the NIH sequencing reads archive with the identifier PRJNA541842. The mouse transcriptomic data has been previously published $7,31,32$ and are available from the GEO repository with the accession codes GSE42533 and GSE104397. The human breast (https://portal.gdc.cancer.gov/projects/TCGA-BRCA) and lung cancer (https:// portal.gdc.cancer.gov/projects/TCGA-LUAD, https://portal.gdc.cancer.gov/projects/ TCGA-LUSC) datasets are available from the TCGA website ${ }^{3,46}$. All the other data supporting the findings of this study are available within the article and its supplementary information files and from the corresponding author upon reasonable request. A reporting summary for this article is available as a Supplementary Information file.

Received: 4 April 2018 Accepted: 27 June 2019

Published online: 22 July 2019

\section{References}

1. Perou, C. M. et al. Molecular portraits of human breast tumours. Nature 406, 747-752 (2000).

2. Prat, A. et al. Phenotypic and molecular characterization of the claudin-low intrinsic subtype of breast cancer. Breast Cancer Res. 12, R68 (2010)

3. Network, T. C. G. A. Comprehensive molecular portraits of human breast tumours. Nature 490, 61-70 (2012).

4. Curtis, C. et al. The genomic and transcriptomic architecture of 2,000 breast tumours reveals novel subgroups. Nature 486, 346-352 (2012).

5. McFadden, D. G. et al. Mutational landscape of EGFR-, MYC-, and Krasdriven genetically engineered mouse models of lung adenocarcinoma. Proc. Natl Acad. Sci. USA 113, E6409-E6417 (2016)

6. Kwong, L. N. et al. Modeling genomic instability and selection pressure in a mouse model of melanoma. Cell Rep. 19, 1304-1312 (2017).

7. Hollern, D. P. \& Andrechek, E. A genomic analysis of mouse models of breast cancer reveals molecular features of mouse models and relationships to human breast cancer. Breast Cancer Res. 16 R59 (2014).

8. Herschkowitz, J. I. et al. Identification of conserved gene expression features between murine mammary carcinoma models and human breast tumors. Genome Biol. 8, R76 (2007).

9. Pfefferle, A. D. et al. Transcriptomic classification of genetically engineered mouse models of breast cancer identifies human subtype counterparts. Genome Biol. 14, R125 (2013).

10. Rennhack, J., To, B., Wermuth, H. \& Andrechek, E. R. Mouse models of breast cancer share amplification and deletion events with human breast cancer. $J$ Mammary Gland Biol. Neoplasia 22, 71-84 (2017).

11. Ben-David, U. et al. The landscape of chromosomal aberrations in breast cancer mouse models reveals driver-specific routes to tumorigenesis. Nat. Commun. 7, 12160 (2016).

12. Guy, C. T. et al. Expression of the neu protooncogene in the mammary epithelium of transgenic mice induces metastatic disease. Proc. Natl Acad. Sci. USA 89, 10578-10582 (1992).

13. Guy, C. T., Cardiff, R. D. \& Muller, W. J. Induction of mammary tumors by expression of polyomavirus middle $\mathrm{T}$ oncogene: a transgenic mouse model for metastatic disease. Mol. Cell Biol. 12, 954-961 (1992)

14. Yeo, S. K., Wen, J., Chen, S. \& Guan, J. L. Autophagy differentially regulates distinct breast cancer stem-like cells in murine models via EGFR/Stat3 and Tgfbeta/Smad signaling. Cancer Res. 76, 3397-3410 (2016).

15. Andrechek, E. R. et al. Genetic heterogeneity of Myc-induced mammary tumors reflecting diverse phenotypes including metastatic potential. Proc. Natl Acad. Sci. USA 106, 16387-16392 (2009).

16. Lawrence, M. S. et al. Mutational heterogeneity in cancer and the search for new cancer-associated genes. Nature 499, 214-218 (2013).

17. Siegel, P. M., Ryan, E. D., Cardiff, R. D. \& Muller, W. J. Elevated expression of activated forms of Neu/ErbB-2 and ErbB-3 are involved in the induction of mammary tumors in transgenic mice: implications for human breast cancer. EMBO J. 18, 2149-2164 (1999).

18. Ma, J. et al. Characterization of mammary cancer stem cells in the MMTVPyMT mouse model. Tumor Biol. 33, 1983-1996 (2012).

19. Langfelder, P. \& Horvath, S. WGCNA: an R package for weighted correlation network analysis. BMC Bioinforma. 9, 559 (2008).
20. Larson, M. H. et al. CRISPR interference (CRISPRi) for sequence-specific control of gene expression. Nat. Protoc. 8, 2180-2196 (2013).

21. Price, J. E. Metastasis from human breast cancer cell lines. Breast Cancer Res. Treat. 39, 93-102 (1996).

22. Nik-Zainal, S. et al. Mutational processes molding the genomes of 21 breast cancers. Cell 149, 979-993 (2012).

23. Alexandrov, L. B. et al. Signatures of mutational processes in human cancer. Nature 500, 415-421 (2013)

24. Nik-Zainal, S. \& Morganella, S. Mutational signatures in breast cancer: the problem at the DNA level. Clin. Cancer Res. 23, 2617-2629 (2017).

25. Rowson-Hodel, A. R. et al. Membrane Mucin Muc4 promotes blood cell association with tumor cells and mediates efficient metastasis in a mouse model of breast cancer. Oncogene 37, 197-207 (2018).

26. Minn, A. J. et al. Genes that mediate breast cancer metastasis to lung. Nature 436, 518-524 (2005).

27. Permuth-Wey, J. et al. Identification and molecular characterization of a new ovarian cancer susceptibility locus at 17q21.31. Nat. Commun. 4, 1627 (2013).

28. Leir, S. H. \& Harris, A. MUC6 mucin expression inhibits tumor cell invasion. Exp. cell Res. 317, 2408-2419 (2011).

29. Yao, Z. et al. A global analysis of the receptor tyrosine kinase-protein phosphatase interactome. Mol. Cell 65, 347-360 (2017).

30. Andrechek, E. R. et al. Amplification of the neu/erbB-2 oncogene in a mouse model of mammary tumorigenesis. Proc. Natl Acad. Sci. USA 97, 3444-3449 (2000).

31. Hollern, D. P., Honeysett, J., Cardiff, R. D. \& Andrechek, E. R. The E2F transcription factors regulate tumor development and metastasis in a mouse model of metastatic breast cancer. Mol. Cell. Biol. 34, 3229-3243 (2014).

32. Andrechek, E. R. HER2/Neu tumorigenesis and metastasis is regulated by E2F activator transcription factors. Oncogene 34, 217-225 (2015).

33. Gatza, M. L. et al. A pathway-based classification of human breast cancer. Proc. Natl Acad. Sci. USA 107, 6994-6999 (2010).

34. Bolger, A. M., Lohse, M. \& Usadel, B. Trimmomatic: a flexible trimmer for Illumina sequence data. Bioinformatics 30, 2114-2120 (2014).

35. Li, H. Aligning sequence reads, clone sequences and assembly contigs with BWA-MEM. Preprint at https://arxiv.org/abs/1303.3997 1303.3997v1 (2013).

36. McKenna, A. et al. The genome analysis toolkit: a mapreduce framework for analyzing next-generation DNA sequencing data. Genome Res. 20, 1297-1303 (2010).

37. Cibulskis, K. et al. Sensitive detection of somatic point mutations in impure and heterogeneous cancer samples. Nat. Biotechnol. 31, 213-219 (2013).

38. Saunders, C. T. et al. Strelka: accurate somatic small-variant calling from sequenced tumor-normal sample pairs. Bioinformatics 28, 1811-1817 (2012).

39. Larson, D. E. et al. SomaticSniper: identification of somatic point mutations in whole genome sequencing data. Bioinformatics 28, 311-317 (2012).

40. Keane, T. M. et al. Mouse genomic variation and its effect on phenotypes and gene regulation. Nature 477, 289-294 (2011).

41. Rausch, T. et al. DELLY: structural variant discovery by integrated paired-end and split-read analysis. Bioinformatics 28, i333-i339 (2012).

42. Krzywinski, M. et al. Circos: an information aesthetic for comparative genomics. Genome Res. 19, 1639-1645 (2009).

43. Ardin, M. et al. MutSpec: a Galaxy toolbox for streamlined analyses of somatic mutation spectra in human and mouse cancer genomes. BMC Bioinformatics 17, 170 (2016).

44. Ran, F. A. et al. Genome engineering using the CRISPR-Cas9 system. Nat Protoc. 8, 2281-2308 (2013)

45. Gilbert, L. A. et al. Genome-scale CRISPR-mediated control of gene repression and activation. Cell 159, 647-661 (2014).

46. Campbell, J. D. et al. Distinct patterns of somatic genome alterations in lung adenocarcinomas and squamous cell carcinomas. Nat. Genet. 48, 607-616 (2016).

47. Gyorffy, B. et al. An online survival analysis tool to rapidly assess the effect of 22,277 genes on breast cancer prognosis using microarray data of 1,809 patients. Breast Cancer Res. Treat. 123, 725-731 (2010).

\section{Acknowledgements}

We thank the members of the Andrechek laboratory for helpful discussions. We thank the Michigan State Investigative HistoPathology Laboratory for the assistance with staining. This work was supported in part by Michigan State University through computational resources provided by the Institute for Cyber-Enabled Research. This work was supported with NIH R01CA160514 and Worldwide Cancer Research WCR - 141153 to E.R.A as well as NIH 1F99CA212221-01 to J.P.R.

\section{Author contributions}

J.R. and E.A. collaborated on the study conception, design, and interpretation of results M.S. provided annotation for translocations and $\mathrm{CN}$ analysis. Y.Z. assisted with copy number validation. C.L., E.B., M.J., C.D., and W.H. provided assistance with in vitro 
experiments. M.P.O., B.T., and S.Y.L. provided assistance with tail vein injections. B.T. contributed to graphic design of Fig. 1A. C.R., K.S., and K.H. collected samples and performed WES. K.H. assisted in the writing of the manuscript and WES study design. J.R. performed all other experiments and drafted the manuscript. All authors have critically read, edited, and approved the final version of the manuscript.

\section{Additional information}

Supplementary Information accompanies this paper at https://doi.org/10.1038/s41467019-11236-3.

Competing interests: The authors declare no competing interests.

Reprints and permission information is available online at http://npg.nature.com/ reprintsandpermissions/

Peer review information: Nature Communications thanks Philip Hinds and other anonymous reviewer(s) for their contribution to the peer review of this work. Peer reviewer reports are available.
Publisher's note: Springer Nature remains neutral with regard to jurisdictional claims in published maps and institutional affiliations.

(c) (i) Open Access This article is licensed under a Creative Commons Attribution 4.0 International License, which permits use, sharing, adaptation, distribution and reproduction in any medium or format, as long as you give appropriate credit to the original author(s) and the source, provide a link to the Creative Commons license, and indicate if changes were made. The images or other third party material in this article are included in the article's Creative Commons license, unless indicated otherwise in a credit line to the material. If material is not included in the article's Creative Commons license and your intended use is not permitted by statutory regulation or exceeds the permitted use, you will need to obtain permission directly from the copyright holder. To view a copy of this license, visit http://creativecommons.org/ licenses/by/4.0/.

(C) The Author(s) 2019 